Case Report

\title{
A Case in Which Angioscopy Was Useful for the Evaluation of Plaque Protrusion during Carotid Artery Stenting
}

Hiroshi Kondo, ${ }^{1,2}$ Yoshihiro Kiura, ${ }^{1,2}$ Shigeyuki Sakamoto, ${ }^{2}$ Takahito Okazaki, ${ }^{2}$ Daizo Ishii, ${ }^{2}$ Katsuhiro Shinagawa, ${ }^{2}$ Nobuhiko Ichinose, ${ }^{2}$ Atsushi Tominaga, ${ }^{1}$ and Kaoru Kurisu ${ }^{2}$

Objective: A case in which angioscopy was useful for the evaluation of plaque protrusion during carotid artery stenting (CAS) is reported.

Case Presentations: The patient was a 67-year-old man. He presented with transient ischemic attacks and progression of bilateral internal carotid artery stenosis during a course of about 7 months. CAS was performed with dual protection using flow reversal system (modified Parodi method) and FilterWire EZ (Boson Scientific, Natick, MA, USA). After stent placement, plaque protrusion was detected by intravascular ultrasonography (IVUS) and optimal coherence tomography (OCT), and plaque protrusion and fluttering debris were also noted by angioscopy. Therefore, additional stenting was performed. Although the evaluation was difficult by subsequent IVUS or OCT, angiosopy revealed plaque protrusion and fluttering debris were both confirmed to be immobilized by the stents.

Conclusion: Since the vascular and stent lumens could be evaluated directly by the use of angioscopy, it may be useful for the examination of plaques and plaque protrusions particularly when multiple stents have been used.

Keywords $>$ angioscopy, carotid artery stenting, plaque protrusion, intravascular ultrasonography,

optimal coherence tomography

\section{Introduction}

In carotid artery stenting (CAS), assessment of plaque morphology is important to evaluate the risk of ischemic complications. In addition to preprocedural evaluation by carotid ultrasonography and MRI, intraprocedural evaluation using intravascular ultrasonography (IVUS) ${ }^{1,2)}$ and optimal coherence tomography $(\mathrm{OCT})^{3,4)}$ have been recently reported. However, imaging using these modalities is indirect. In this study, we directly examined the vascular lumen

${ }^{1}$ Department of Neurosurgery and Neuroendovascular Therapy, Hiroshima Prefectural Hospital, Hiroshima, Hiroshima, Japan ${ }^{2}$ Department of Neurosurgery, Graduate School of Biomedical and Health Sciences, Hiroshima University, Hiroshima, Hiroshima, Japan

Received: October 16, 2016; Accepted: May 21, 2017

Corresponding author: Hiroshi Kondo. Department of Neurosurgery and Neuroendovascular Therapy, Hiroshima Prefectural Hospital, 1-5-54 Ujinakanda, Minami-ku, Hiroshima, Hiroshima 734-0004, Japan

Email: springhascome0224@gmail.com

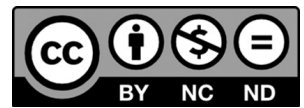

This work is licensed under a Creative Commons Attribution-NonCommercialNoDerivatives International License.

(C)2017 The Japanese Society for Neuroendovascular Therapy by angioscopy in a patient who underwent CAS for cervical internal carotid artery (ICA) stenosis.

\section{Angioscopy system}

The angioscopy system used in this study has been approved for coronary artery disease, but not for carotid artery disease in Japan. Therefore, we used it with approval by the institutional review board of our hospital. VISIBLE (FiberTech Co., Ltd, Tokyo, Japan) with an inserted part $0.75 \mathrm{~mm}$ in external diameter and $1620 \mathrm{~mm}$ in usable length was used as a three charge-coupled device (3CCD) angioscopic catheter, and FT-203F (FiberTech Co.) was used as a 3CCD imaging system. The common carotid artery (CCA) on the proximal side was occluded with a balloon guiding catheter, and the external carotid artery (ECA) was occluded with a balloon protection system. After a filter protection device was deployed in the ICA, VISIBLE was inserted into the aspiration catheter and navigated to the carotid lesion (Fig. 1). Then, the ICA blood flow was blocked by inflating both the balloon of the guiding catheter in CCA and occlusion balloon in ECA, intraluminal blood was replaced by injection $50 \mathrm{~mL}$ of saline at $6-8 \mathrm{~mL} / \mathrm{sec}$ through the guiding catheter, and the vascular lumen was observed for 6-8 seconds. 

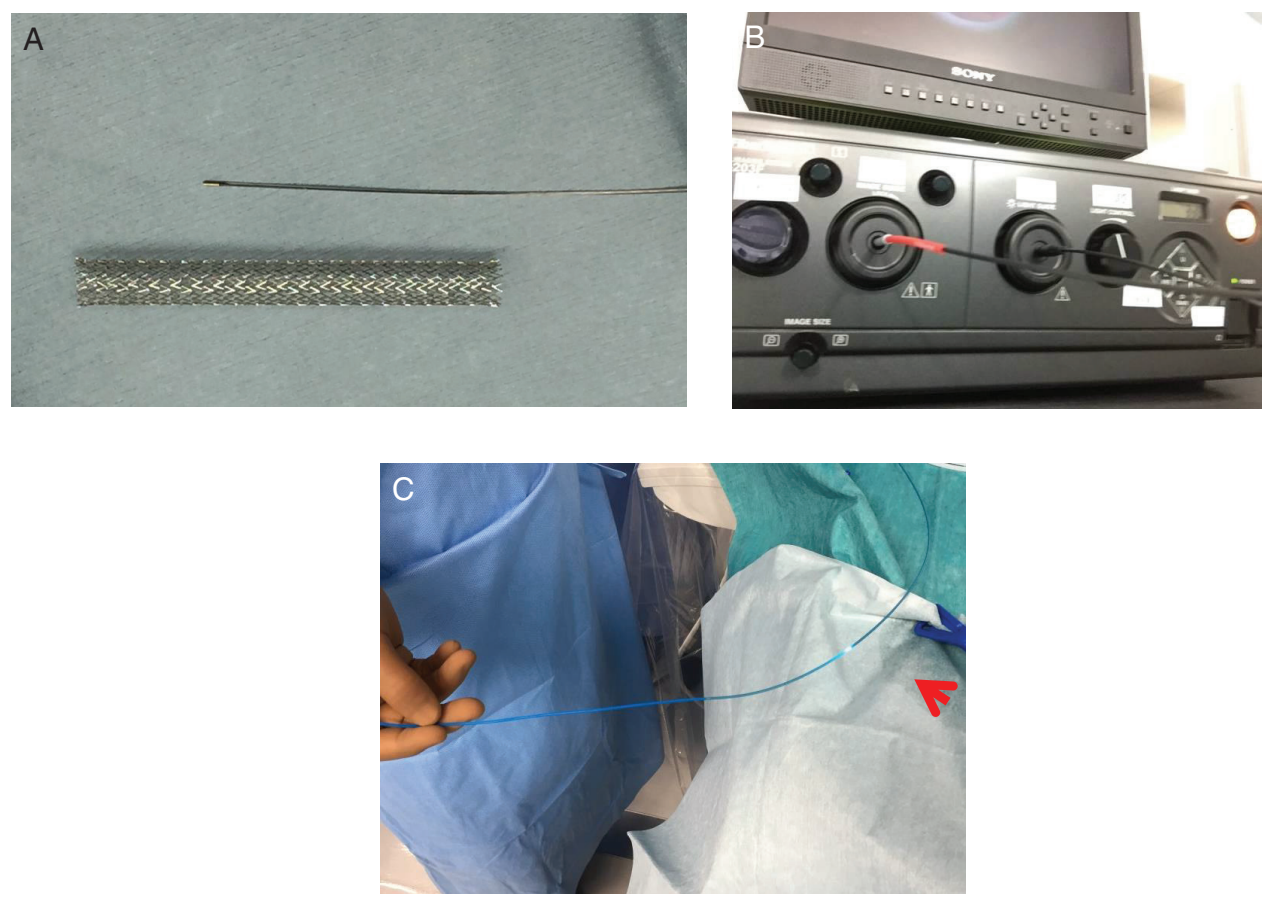

Fig. 1 (A) 3 CCD angioscopic catheter (VISIBLE) and Carotid Wallstent $(10 \mathrm{~mm} \times 80 \mathrm{~mm})$. (B) 3 CCD imaging system (FT-203F). (C) VISIBLE in the aspiration catheter (EXPORT). CCD: charge-coupled device

\section{Case Presentation}

The patient was a 67-year-old man with a history of dyslipidemia. While he was followed up for bilateral ICA stenosis, a revascularization therapy was considered necessary as he developed transient ischemic attach (left hemiplegia), MRA demonstrated marked progression of bilateral ICA stenosis over a period of about 7 months, and signals intensity of the right intracranial arteries were decreased. On MRI plaque imaging, the plaques appeared as high signal intensity areas in T1-weighted images, and unstable plaques were suspected (Fig. 2).

\section{Carotid artery stenting}

As preprocedural antiplatelet therapy, the administration of aspirin $100 \mathrm{mg}$, clopidogrel $75 \mathrm{mg}$, and cilostazol $200 \mathrm{mg}$ per day was continued. The patient was systemically heparinized during the procedure, and the activated clotting time (ACT) was maintained at $\geq 275 \mathrm{sec}$.

A 9 French ( $\mathrm{Fr}$ ) long sheath was placed in the right femoral artery, and a 9 Fr balloon guiding catheter (OPTIMO; Tokai Medical Product, Aichi, Japan) was placed in the CCA using a 6 Fr inner catheter (JB2; Medikit, Tokyo, Japan) and a 0.035 inch stiff guidewire (Radifocus Guidewire M Stiff type; Terumo Interventional Systems, Tokyo, Japan). As a flow reversal system, a 4 Fr sheath was placed in the left femoral vein and connected to the OPTIMO via a filter. A Carotid GuardWire (Medtronic Inc., Minneapolis, $\mathrm{MN}$ ) was navigated in the ECA to the proximal side of the superior thyroid artery, and a FilterWire EZ (Boson Scientific, Natick, MA, USA) was advanced through the lesion and deployed in the ICA. Before the stenting, IVUS, OCT, and angioscopy were performed once. Then, the OPTIMO and Carotid Guardwire were inflated to establish a state of flow reversal, pre-dilatation was performed using a Jackal (Kaneka, Osaka, Japan) $3.0 \times 30 \mathrm{~mm}$, and a Carotid Wallstent (Boston Scientific) $8 \mathrm{~mm} \times 21 \mathrm{~mm}$ was deployed. Post-dilatation was performed using a Jackal $5.0 \times 30 \mathrm{~mm}$ (Fig. 3A), and aspiration using an Export aspiration catheter (Medtronic Inc.) was performed. Plaque protrusion was detected by IVUS and OCT after stent placement (Fig. 3B and $\mathbf{3 C}$ ). When the angioscopy was inserted into the Export and navigated to the lesion, plaque protrusion and fluttering debris protruding inside the stent were confirmed (Fig. 3D and $3 E$ ). Therefore, an additional stent of the same size (Carotid Wallstent $8 \times 21 \mathrm{~mm}$ ) was placed (Fig. 4A). IVUS and OCT were performed again. While IVUS showed no clear plaque protrusion, contrast was strong due to the presence of two stents, and the evaluation was considered difficult (Fig. 4B). On OCT, partial plaque protrusion was noted unlike the IVUS finding (Fig. 4C). Angioscopy was performed, and since the plaque protrusion and fluttering 

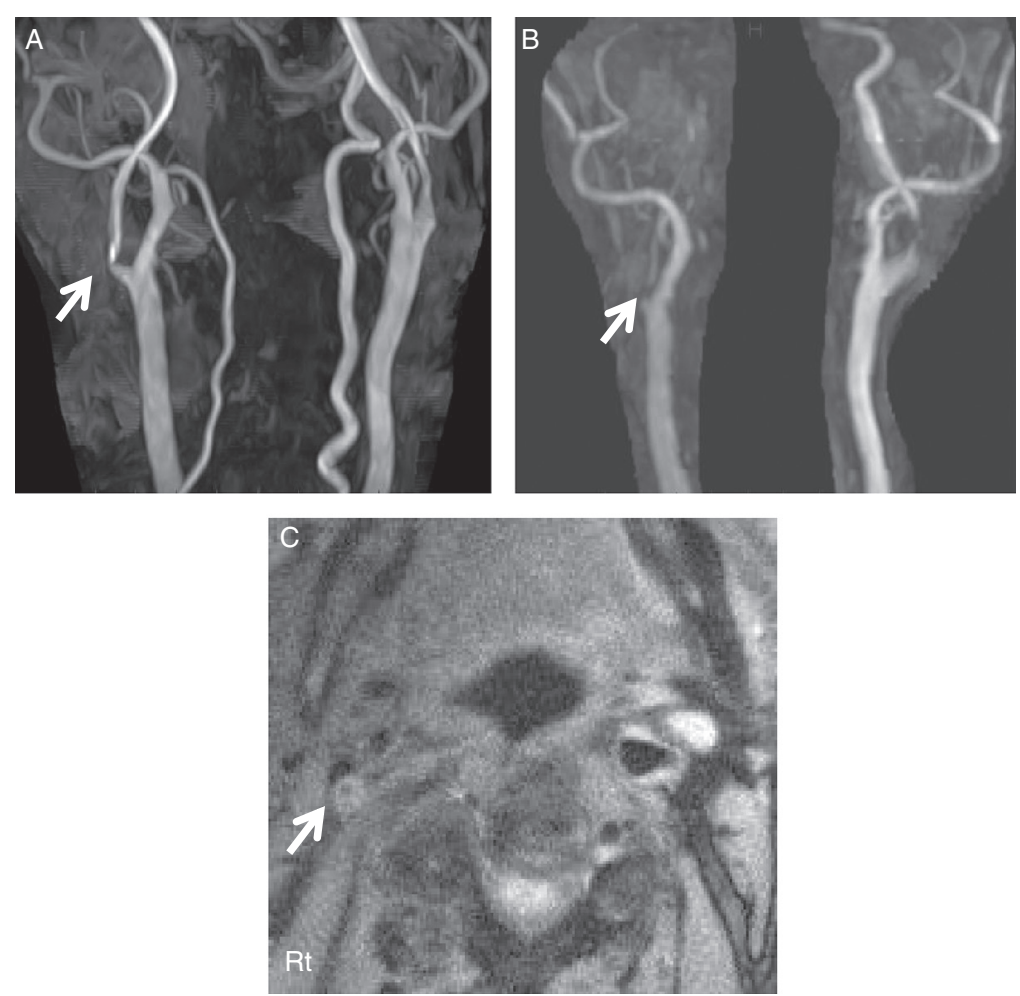

Fig. 2 MRA showed bilateral ICA stenosis (A) and after 7 months, MRA revealed bilateral ICA stenosis progression (B). The plaque was thought to be vulnerable by T1 plaque image (C). ICA: internal carotid artery

debris observed previously were sufficiently controlled (Fig. 4D and 4E), the procedure was ended. Captured debris was found in the FilterWire EZ.

Postprocedural MRI showed a small high intensity spot on diffusion-weighted imaging (DWI) at one site, no neurologic deficits were observed.

\section{Discussion}

CAS became a treatment with evidence for patients with cervical carotid artery stenosis at a high risk for carotid artery endarterectomy (CEA) after the Stenting and Angioplasty with Protection in Patients at High Risk for Endarterectomy study (SAPPHIRE) was reported in 2004.5) Thereafter, as the Carotid Revascularization Endarterectomy vs Stenting trial (CREST) was published, indications for CAS were also expanded to the standard risk group for CEA. However, cerebral infarction as a periprocedural complication is reported to occur more frequently in the CAS group (4.1\%) than in the CEA group (2.3\%). ${ }^{6}$ In addition, despite the difference in the embolic protection device (EPD), the periprocedural DWI-positive rate after CAS has been reported to be $17.3 \%-64 \% .^{7-10)}$ Since a relationship between the plaque characteristics and ischemic complications has been suggested, ${ }^{11)}$ the pre- and intraprocedural of plaque morphology assessment is important. There have been reports of preprocedural evaluation using carotid ultrasonography and MRI, and the presence of vulnerable plaque is considered as a risk factor of periprocedual cerebral infarction. ${ }^{12-14)}$

The use of IVUS $^{2}$ and $\mathrm{OCT}^{3)}$ for the intraprocedural assessment of plaques and the interior of the stent has been reported. As for angioscopy, by which the interior of the stent can be observed directly, there have been reports since the 1980 s concerning the coronary artery and peripheral arteries. ${ }^{15-17)}$ In the coronary artery, also, a new assessment technique using Evans blue has recently been reported in addition to the diagnosis according to the color of plaques observed by angioscopy. ${ }^{18,19)}$ These techniques can evaluate the plaque characteristics in real time, as well as detection of plaque protrusion after stent placement. However, angioscopy needs the injection of saline which can increase the risk of distal embolism during CAS. The frequency of the appearance of plaque protrusion after CAS was reported 7.8\% using IVUS by Shinozaki et al., ${ }^{1)}$ and $17.6 \%$ using OCT according to the evaluation by Yoshimura 


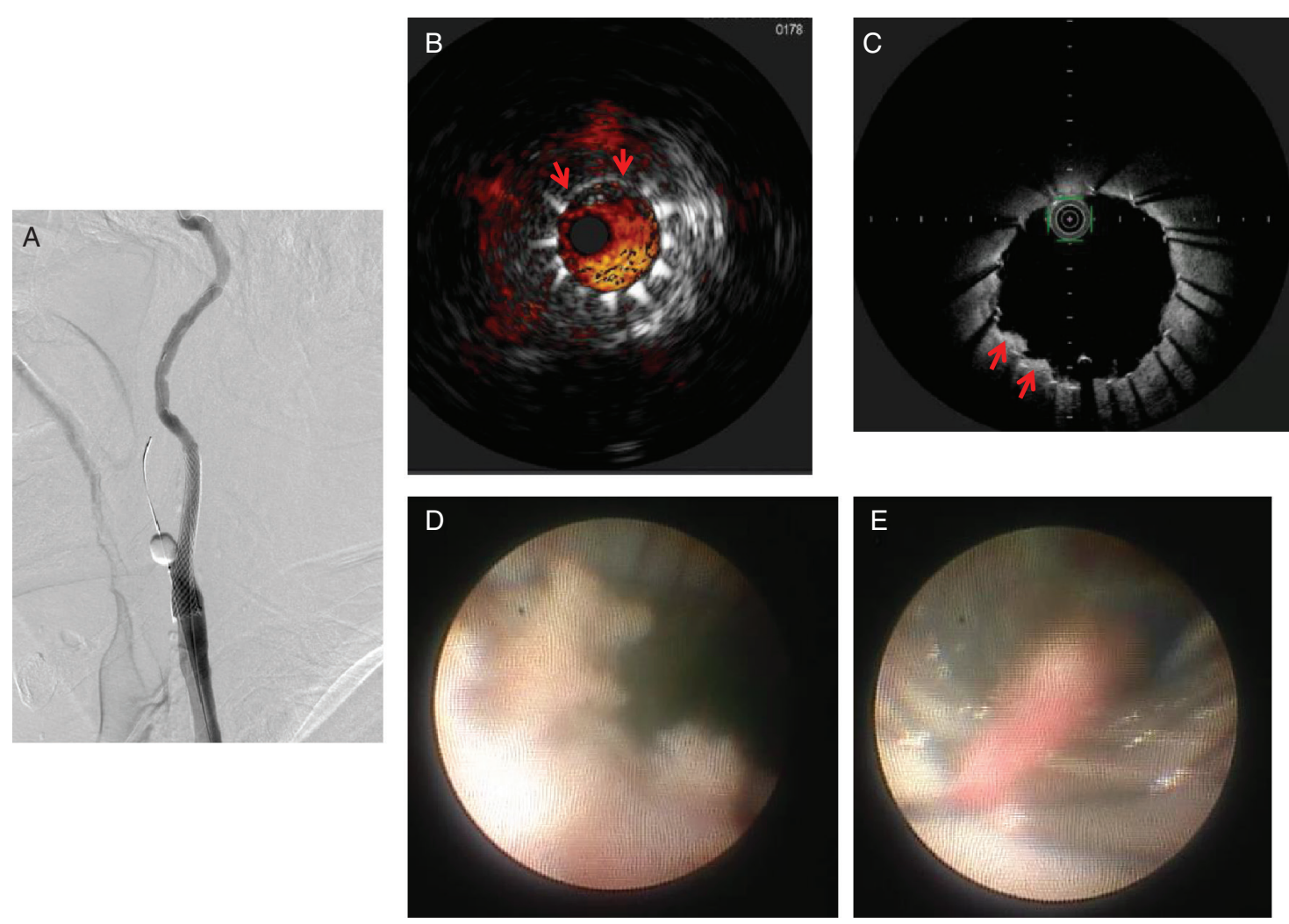

Fig. 3 (A) DSA after first stent (Carotid Wallstent $8 \mathrm{~mm} \times 21 \mathrm{~mm}$ ) placement. Plaque protrusion was revealed by IVUS (B), and OCT (C). Angioscopy showed plaque protrusion (D) and fluttering debris (E) in the Carotid Wallstent. IVUS: intravascular ultrasonography; OCT: optimal coherence tomography

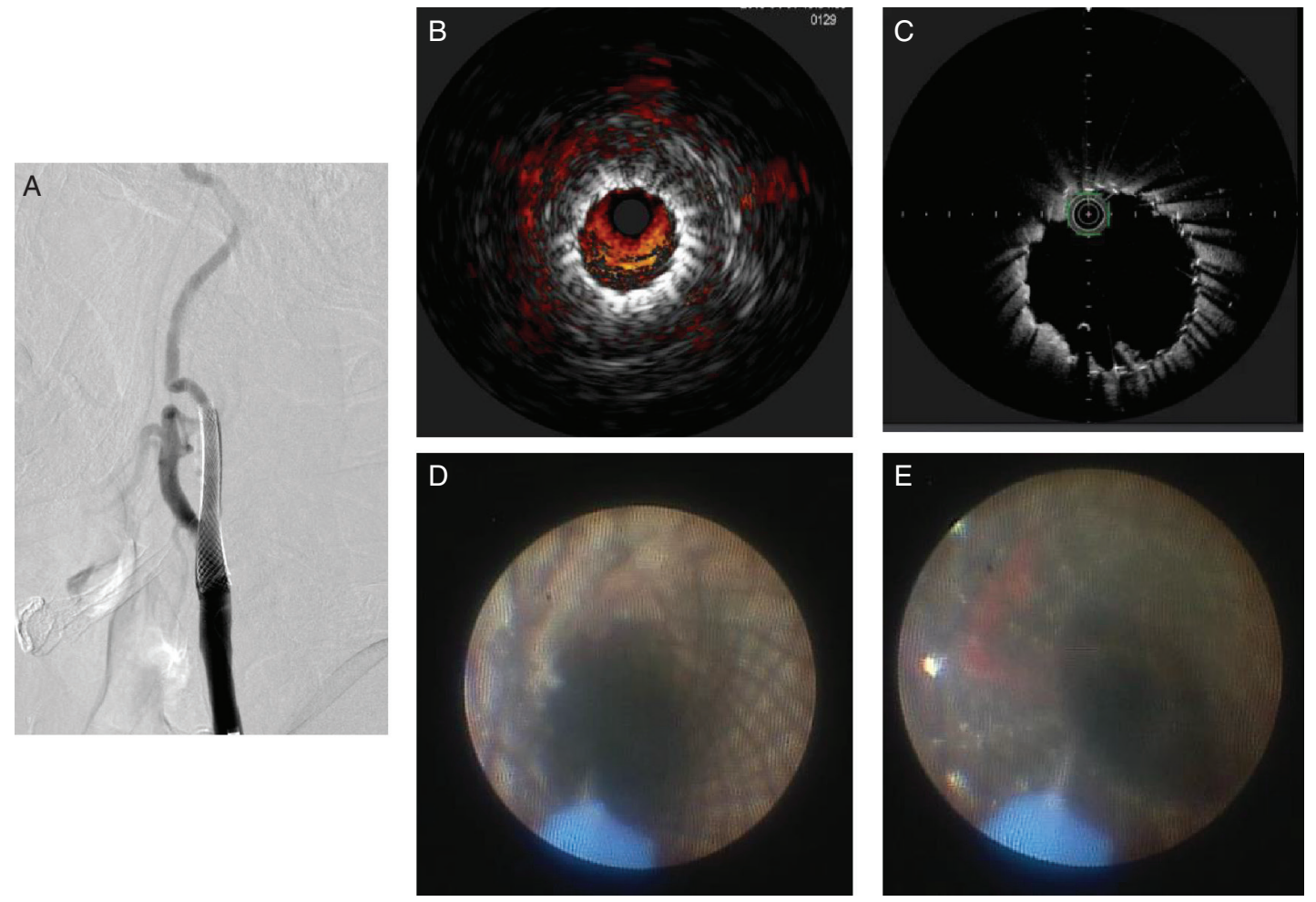

Fig 4 (A) DSA after additional stent (Carotid Wallstent $8 \mathrm{~mm} \times 21 \mathrm{~mm}$ ) placement. IVUS (B) could not describe any remarkable abnormal finding and OCT (C) showed plaque protrusion. Angioscopy revealed the additional stent suppressed the plaque protrusion (D) and fluttering debris (E). IVUS: intravascular ultrasonography; OCT: optimal coherence tomography 
et al. ${ }^{4)}$ It was highest at $25 \%$ in the report by Tanemura et al.,20) who directly examined the interior of the stent by angioscopy. Also, as fluttering plaques as observed in our patient are difficult to detect by IVUS or OCT, angioscopy is considered effective for their detection. Moreover, angioscopy considered can also be considered useful in patients who have undergone additional stent placement as our patient did since evaluation by IVUS may be difficult due to contrast.

Concerning the carotid artery, Tanemura et al. ${ }^{20)}$ reported the results of angioscopy during CAS in 18 patients. In this report, clear views were obtained in 12 patients. Of those in whom clear views could not be obtained, four patients were reported to have problems with the removal of blood due to incomplete obstruction. In our patient, the CCA and ECA of the proximal side of the superior thyroid artery could be occluded, and reflux of blood from the ICA was relatively small, resulting in satisfactory removal of blood and a clear view. Some measures to achieve complete blood removal are also considered necessary. While no symptomatic ischemic complications were observed in the report by Tanemura et al., ${ }^{20)}$ the DWI-positive rate was relatively high at $47 \%$. One reason for this high DWI-positive rate may due to distal protection of the ICA using Carotid Guardwire alone or the use of the Parodi antiemboli system (PAES) with no distal protection for the ICA. While DWI hyperintensity was positive in our patient, Sakamoto et al. ${ }^{10)}$ reported that the DWI positive rate can be reduced by dual protection (proximal and distal protection), suggesting the possibility of its further reduction by a similar method.

Regarding problems with angioscopy other than blood removal is the restriction of the observable area due to the narrow angle of the visual field, while the nearly entire vascular lumen could be examined in the present case.

\section{Conclusion}

In CAS, angioscopy, which provides a clear view and makes direct observation of the vascular or stent lumen possible, is considered very useful for the detection of plaques and plaque protrusion. Particularly, it can be useful for direct examination of fluttering plaques, which are usually undetectable by IVUS or OCT.

\section{Disclosure Statement}

The first author and all of the coauthors have no conflicts of interest.

\section{References}

1) Shinozaki N, Ogata N, Ikari Y: Plaque protrusion detected by intravascular ultrasound during carotid artery stenting. J Stroke Cerebrovasc Dis 2014; 23: 2622-2625.

2) Hitchner E, Zayed MA, Lee G, et al: Intravascular ultrasound as a clinical adjunct for carotid plaque characterization. J Vasc Surg 2014; 59: 774-780.

3) Yoshimura S, Kawasaki M, Yamada K, et al: OCT of Human carotid arterial plaques. JACC Cardiovasc Imaging 2011; 4: 432-436.

4) Yoshimura S, Kawasaki M, Yamada K, etal:Optical Coherence Tomography (OCT): A new Imaging tool during carotid artery stenting. In: Kawasaki M, eds. optical coherence tomography. IN TECH; 2013:Chapter 6. https://www.intechopen.com/ books/optical-coherence-tomography/optical-coherencetomography-oct-a-new-imaging-tool-during-carotidartery-stenting. (Accessed October 2, 2016).

5) Yadav JS, Wholey MH, Kuntz RE, et al: Protected carotidartery stenting versus endarterectomy in high-risk patients. N Engl J Med 2004; 351: 1493-1501.

6) Brott TG, Hobson RW, Howard G, et al: Stenting versus endarterectomy for treatment of carotid-artery stenosis. N Engl J Med 2010; 363: 11-23.

7) Koichi S, Mami H, Tetsuya T, et al: [Transition of carotid artery stenting treatment in the Multiple Device Era (effectiveness of distal balloon protection CAS treatment using an Autologous Aspirated Blood Transfusion)]. Surg Cereb Stroke 2015; 43: 347-351. (in japanese)

8) Schnaudigel S, Gröschel K, Pilgram SM, et al: New brain lesions after carotid stenting versus carotid endarterectomy: a systematic review of the literature. Stroke 2008; 39: 1911-1919.

9) Piñero P, González A, Mayol A, et al: Silent ischemia after neuroprotected percutaneous carotid stenting: a diffusionweighted MRI study. AJNR Am J Neuroradiol 2006; 27: 1338-1345.

10) Sakamoto S, Kiura $Y$, Okazaki $T$, et al: Usefulness of dual protection combined with blood aspiration for distal embolic protection during carotid artery stenting. Acta Neurochir (Wien) 2015; 157: 371-377.

11) Yamada K, Yoshimura S, Kawasaki M, et al: Embolic complications after carotid artery stenting or carotid endarterectomy are associated with tissue characteristics of carotid plaques evaluated by magnetic resonance imaging. Atherosclerosis 2011; 215: 399-404.

12) Kobayashi E, Ono J, Hirai S, et al: Detection of unstable plaques in patients with carotid stenosis using B-mode ultrasonography. Interv Neuroradiol 2000; 6: 165-170.

13) Masaomi K, Kazumichi $Y$, Natuse K, et al: [Carotid artery stenting based on plaque characteristics using magnetic resonance plaque imaging]. JNET 2012; 6: 240-244. (in Japanese) 
14) Yoshimura S, Yamada K, Kawasaki M, et al: Highintensity signal on time-of-flight magnetic resonance angiography indicates carotid plaques at high risk for cerebral embolism during stenting. Stroke 2011; 42: 3132-3137.

15) Spears JR, Marais HJ, Serur J, et al: In vivo coronary angioscopy. J Am Coll Cardiol 1983; 1: 1311-1314.

16) Mizuno $K$, Arai $T$, Satomura $K$, et al: New percutaneous transluminal coronary angioscope. JACC 1989; 13: 363-368
17) Uchida $Y$, Tomaru $T$, Nakamura F, et al: Percutaneous coronary angioscopy in patients with ischemic heart disease. Am Heart J 1987; 114: 1216-1222.

18) Uchida Y: Recent advances in coronary angioscopy. J Cardiol 2011; 57: 18-30.

19) Uchida $Y$, Uchida Y: Dye-staining angioscopy for coronary artery disease. Curr Cardiovasc Imaging Rep 2015; 8: 10.

20) Tanemura H, Hatazaki S, Asakura F, et al: Angioscopic observation during carotid angioplasty with stent placement. AJNR Am J Neuroradiol 2005; 26: 1943-1948. 